\title{
ESTRESSE OCUPACIONAL E ENGAJAMENTO EM PROFISSIONAIS DE SAÚDE BUCAL
}

\section{Occupational stress and engagement in oral health professionals}

\section{Estrés laboral y compromiso de profesionales de salud bucal}

\author{
Jussara Rossi Castro iD \\ Faculdade de Medicina de São José do Rio Preto - FAMERP - São José do Rio Preto (SP) - Brasil \\ Cláudia Eli Gazetta iD \\ Faculdade de Medicina de São José do Rio Preto - FAMERP - São José do Rio Preto (SP) - Brasil \\ Albertina Gomes da Silva (iD \\ Faculdade de Medicina de São José do Rio Preto - FAMERP - São José do Rio Preto (SP) - Brasil \\ Paula Canova Sodré (iD \\ Faculdade de Medicina de São José do Rio Preto - FAMERP - São José do Rio Preto (SP) - Brasil \\ Luciano Garcia Lourenção (iD \\ Universidade Federal do Rio Grande - FURG - Rio Grande (RS) - Brasil
}

\section{RESUMO}

Objetivo: Avaliar os níveis de estresse ocupacional e engajamento em profissionais de saúde bucal de unidades da Atenção Primária à Saúde. Métodos: Estudo quantitativo, descritivo, correlacional e transversal, realizado com profissionais da saúde bucal das unidades de Atenção Primária à Saúde do município de São José do Rio Preto, São Paulo, em 2017. Utilizaram-se três instrumentos autoaplicáveis: um elaborado pelos pesquisadores, contendo variáveis sociodemográficas e profissionais, a Escala de Estresse no Trabalho e a Utrecht Work Engagement Scale. Os níveis de estresse ocupacional e engajamento foram calculados segundo modelo estatístico proposto pelos instrumentos e, para a análise, utilizou-se teste de correlação de Pearson, considerando significância de 95\%. Resultados: Participaram 31 profissionais, sendo $20(64,5 \%)$ cirurgiões-dentistas e 11 (35,5\%) auxiliares de saúde bucal. Houve prevalência do sexo feminino ( $n=21 ; 67,7 \%)$, faixa etária de 40 anos ou mais $(n=14 ; 45,2 \%)$, casados $(n=26 ; 83,9 \%)$, concursados/estatutários $(n=16 ; 51,6 \%)$, com carga horária semanal de 40 horas $(n=23 ; 74,2 \%)$, sem outra atividade remunerada $(n=19 ; 61,3 \%)$, três a dez anos de atuação na Atenção Primária à Saúde $(n=13 ; 41,9 \%)$ e satisfeitos com a profissão $(n=28 ; 90,3 \%)$. A amostra não apresentou níveis importantes de estresse, mas nove $(29,0 \%)$ profissionais obtiveram escores compatíveis com estresse importante $(\geq 2,5)$. Os profissionais apresentaram níveis altos de engajamento em todas as dimensões. Estresse ocupacional e engajamento se correlacionam negativamente. Conclusão: Os trabalhadores apresentaram altos níveis de engajamento. Estresse ocupacional e engajamento tendem a ser inversamente proporcionais.

Descritores: Estresse Ocupacional; Engajamento no Trabalho; Odontólogos; Auxiliares de Odontologia; Atenção Primária à Saúde.

\section{ABSTRACT}

Objective: To evaluate the levels of occupational stress and engagement in oral health professionals from Primary Health Care units. Methods: It is a quantitative, descriptive, correlational and cross-sectional study conducted with oral health professionals from Primary Health Care units in the city of São José do Rio Preto, São Paulo, in 2017. We used three self-applied instruments: one developed by the researchers, containing sociodemographic and professional variables, the Stress at Work Scale, and the Utrecht Work Engagement Scale. Occupational stress and engagement levels were calculated according to the statistical model proposed by the instruments and, for the analysis, Pearson's correlation test was used, considering the significance of $95 \%$. Results: 31 professionals participated, being 20 (64.5\%) dental surgeons and $11(35.5 \%)$ oral health auxiliaries. There was a prevalence of females ( $n=21 ; 67.7 \%)$, aged 40 years or older $(n=14 ; 45.2 \%)$, married $(n=26 ; 83.9 \%)$, hired / statutory $(n=16 ; 51.6 \%)$, with a weekly workload of 40 hours ( $n=23 ; 74.2 \%)$, without any other paid activity $(n=19 ; 61.3 \%)$, three to ten years of experience in the Primary Health Care $(n=13 ; 41.9 \%)$ and satisfied with the profession $(n=28 ; 90.3 \%)$. The sample did not show significant stress levels, but nine (29.0\%) professionals obtained scores compatible with significant stress (> 2.5). Professionals had high

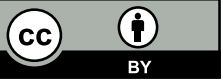


levels of engagement in all dimensions. Occupational stress and engagement correlate negatively. Conclusion: Workers had high levels of engagement. Occupational stress and engagement tend to be inversely proportional.

Descriptors: Occupational Stress; Engagement at Work; Dentists; Dental Auxiliaries; Primary Health Care.

\section{RESUMEN}

Objetivo: Evaluar los niveles de estrés laboral y compromiso de profesionales de salud bucal de unidades de la Atención Primaria de Salud. Métodos: Estudio cuantitativo, descriptivo, de correlación y transversal realizado con profesionales de salud bucal de las unidades de Atención Primaria de Salud del municipio de São José do Rio Preto, São Paulo, en 2017. Se ha utilizado tres instrumentos auto aplicables: uno de ellos ha sido elaborado por los investigadores con variables sociodemográficas y profesionales, la Escala de Estrés en el Trabajo y la Utrecht Work Engagement Scale. Los niveles del estrés laboral y del compromiso han sido calculados según el modelo estadístico propuesto por los instrumentos y para el análisis se utilizó la prueba de correlación de Pearson con significancia del 95\%. Resultados: Participaron 31 profesionales siendo 20 (64,5\%) cirujanos-dentistas y 11 (35,5\%) auxiliares de salud bucal. Hubo prevalencia para el sexo femenino ( $n=21 ; 67,7 \%)$, la franja de edad de 40 años o más $(n=14 ; 45,2 \%)$, casados ( $n=26 ; 83,9 \%)$, concursantes/estatutarios $(n=16 ; 51,6 \%)$, con 40 horas semanales de trabajo ( $n=23$; $74,2 \%)$, sin otra actividad remunerada ( $n=19 ; 61,3 \%)$, entre tres y diez años de actuación en la Atención Primaria de Salud ( $n=13 ; 41,9 \%)$ y satisfechos con la profesión $(n=28 ; 90,3 \%)$. La muestra no ha presentado niveles importantes de estrés pero nueve $(29,0 \%)$ profesionales tuvieron puntuaciones compatibles con el estrés importante $(\geq 2,5)$. Los profesionales presentaron niveles altos de compromiso para todas las dimensiones investigadas. El estrés laboral y el compromiso se han correlacionado de manera negativa. Conclusión: Los trabajadores presentaron altos niveles de compromiso. El estrés laboral y el compromiso tienden a ser inversamente proporcionales.

Descriptores: Estrés Laboral; Compromiso Laboral; Odontólogos; Auxiliares Dentales; Atención Primaria de Salud.

\section{INTRODUÇÃO}

A Estratégia Saúde da Família (ESF), implantada no Brasil na década de 1990, como modelo de reorientação das práticas sanitárias, surgiu na intenção de substituir as antigas práticas de valorização do hospital por propostas focadas na promoção da saúde, tendo a família como principal ponto de atenção, e não o indivíduo doente, de maneira a não esperar os usuários chegarem para serem atendidos, mas agir preventivamente sobre eles a partir de um novo modelo de atenção(1).

Embora a participação dos profissionais de saúde bucal nas atividades da ESF tenha sido proposta desde a sua implantação, a incorporação oficial desses profissionais ocorreu apenas nos anos 2000 , quando o Ministério da Saúde estabeleceu um incentivo financeiro para inclusão das equipes de saúde bucal (eSB) na ESF(2).

De acordo com a Política Nacional de Atenção Básica vigente, as eSB devem ser constituídas por cirurgião-dentista e técnico em saúde bucal (TSB) e/ou auxiliar de saúde bucal (ASB), alocados em uma Unidade Básica de Saúde (UBS), ou Unidade Odontológica Móvel, e vinculados a uma equipe de Atenção Básica (eAB), ou equipe de Saúde da Família (eSF). As eSB são classificadas em duas modalidades: modalidade I, composta por um cirurgião-dentista e um ASB ou TSB; e modalidade II, composta por um cirurgião-dentista, um TSB e um ASB, ou dois TSB ${ }^{(3)}$.

Algumas características inerentes à atividade laboral dos profissionais de saúde bucal, como posições posturais inadequadas, campo operatório mal iluminado, trabalho sob pressão, além da necessidade de lidar com a angustia e a dor do paciente, fazem da profissão uma das mais estressantes na área da saúde ${ }^{(4)}$.

Nesse contexto, a compreensão de fatores estressantes que podem comprometer a saúde dos profissionais de Odontologia, consequentemente, das eSB da ESF, pode ser uma via para melhorias, pois o conhecimento da situação permite a reorganização do sistema, prevenindo agravos à saúde. Podendo-se, ainda, criar estratégias para uma reestruturação que favoreça a melhoria das condições de trabalho, com repercussão no aumento da produtividade e na qualidade da assistência( ${ }^{(5)}$.

Por outro lado, os aspectos positivos da relação dos profissionais de saúde com o ambiente laboral podem fortalecer o vínculo dos trabalhadores com a prática profissional, melhorando a qualidade dos serviços e a resolutividade dos problemas dos usuários. Nesse contexto, o engajamento representa um estado mental positivo relacionado ao trabalho, caracterizado por vigor, dedicação e absorção( ${ }^{(6,7)}$. Foi conceituado, na década de 1990 , como um estado mental afetivo-emocional positivo em relação ao trabalho e indica persistência, engloba energia, envolvimento emocional, dedicação ao trabalho e imersão nas atividades de trabalho(6-8). 
Reforçando esse argumento, alguns estudos enfatizam maiores índices de engajamento profissional relacionado com titulação acadêmica, como uma pesquisa de cirurgiãs-dentistas indianas com título de mestrado que possuíam índices de engajamento maiores que os demais profissionais ${ }^{(9)}$. Outro estudo, realizado na Holanda, para avaliar os níveis de engajamento de cirurgiões dentistas e os recursos odontológicos relacionados, mostraram escores de engajamento relativamente altos ${ }^{(10)}$.

Nesse contexto, conhecer os níveis de engajamento e de estresse ocupacional, bem como identificar os estressores mais importantes na percepção dos profissionais das equipes de saúde bucal poderá contribuir para o direcionamento de ações de melhoria das condições de trabalho e saúde desses profissionais, reduzindo riscos e vulnerabilidades inerentes ao ambiente e ao processo de trabalho, conforme estabelecido pela Política Nacional de Promoção da Saúde ${ }^{(11)}$. Além disso, os resultados poderão contribuir para o aprimoramento das políticas públicas de atenção à saúde dos trabalhadores no município, a partir do fortalecimento das ações de promoção de saúde e qualidade de vida no trabalho.

Ante o exposto, este estudo objetivou avaliar os níveis de estresse ocupacional e engajamento em profissionais de saúde bucal de unidades da Atenção Primária à Saúde.

\section{MÉTODOS}

Estudo quantitativo, descritivo, correlacional e transversal realizado com profissionais de saúde bucal das unidades da atenção primária à saúde do município de São José do Rio Preto, situado na região Noroeste do estado de São Paulo, a 452 km da capital. Possui população estimada em 450.657 habitantes $^{(12)}$.

O Sistema Municipal de Saúde está estruturado em cinco Distritos de Saúde. Conta com 27 unidades da Atenção Básica, sendo 10 Unidades Básicas de Saúde (UBS) e 17 Unidades Básicas de Saúde da Família (UBSF), com 20 equipes de saúde bucal ${ }^{(13)}$.

A população do estudo foi composta por profissionais das eSB (cirurgiões-dentistas e auxiliares de saúde bucal) das unidades de saúde da atenção primária (10 UBS e 17 UBSF) do município de São José do Rio Preto, São Paulo, sendo estimados 40 profissionais. Excluíram-se os profissionais que estavam de férias e/ou afastados das atividades profissionais no período da coleta dos dados.

Utilizaram-se três instrumentos para coleta dos dados: um elaborado pelos autores para coletar informações sobre o perfil sociodemográfico e profissional dos trabalhadores da APS; a Escala de Estresse no Trabalho (EET), validada em estudo ${ }^{(14)}$ realizado em organizações públicas e privadas no Distrito Federal e em São Paulo; e a Utrecht Work Engagement Scale (UWES), validada em estudo ${ }^{(15)}$ realizado nas cinco regiões brasileiras.

A EET é composta por 23 afirmativas negativas, com uma escala de 5 pontos, variando desde " 1 - discordo totalmente" a " 5 - concordo totalmente", sendo que, quanto maior a pontuação, maior o estresse. Os indicadores da EET foram elaborados a partir da análise da literatura sobre estressores organizacionais de natureza psicossocial e sobre reações psicológicas ao estresse ocupacional. A EET consiste numa medida geral de estresse, cujos itens abordam diversos estressores e reações emocionais constantemente associadas a eles. $O$ estudo ${ }^{(14)}$ de validação indica que a EET possui características psicométricas satisfatórias e pode contribuir tanto para pesquisas sobre $o$ tema quanto para o diagnóstico do ambiente organizacional. A EET não é um teste psicológico, mas uma ferramenta para diagnóstico organizacional que foi submetida a testes e requisitos psicométricos ${ }^{(14)}$.

A UWES é composta por dezessete itens de autoavaliação com três dimensões (vigor, dedicação e absorção) e um escore geral ${ }^{(15)}$. A mensuração do vigor consiste na média dos seis itens que se referem aos altos níveis de energia e resiliência, vontade de investir esforços, não se fadigar com facilidade, e persistir face a dificuldades ${ }^{(15,16)}$. A dedicação é mensurada pela média dos cinco itens relacionados a um senso de significado pelo trabalho, sentirse entusiasmado e orgulhoso em relação ao seu labor, sentir-se inspirado e desafiado por ele ${ }^{(15,16)}$.

Para a mensuração da absorção calcula-se a média dos seis itens referentes a estar totalmente imerso em seu trabalho e ter dificuldades em desapegar-se dele, o tempo passa rapidamente e ele esquece-se de tudo ao seu redor ${ }^{(15,16)}$. O escore geral consiste na média de todos os itens da UWES ${ }^{(15,16)}$.

A coleta dos dados foi realizada no período de janeiro a março de 2017. Após autorização da Secretaria Municipal de Saúde, os pesquisadores entraram em contato com os gerentes das unidades de saúde incluídas no estudo para apresentação da pesquisa e de seus objetivos e a assinatura do Termo de Consentimento Livre e Esclarecido (TCLE) pelos participantes.

Os questionários e os TCLE eram entregues aos gerentes, encarregados de distribuí-los para os profissionais do estudo e recebê-los após o preenchimento. Visando a preservar a identidade dos participantes do estudo, os 
instrumentos de coleta dos dados eram recolhidos e armazenados em envelopes separados dos TCLE. Em seguida, os envelopes com os instrumentos respondidos eram entregues aos pesquisadores.

Armazenaram-se os dados obtidos em um banco de dados, utilizando-se a planilha do programa Microsoft Excel $囚$, para possibilitar a análise de acordo com os objetivos propostos. Utilizou-se o programa Statistical Package for Social Sciences (SPSS), versão 20.0, na análise dos dados.

As variáveis sociodemográficas foram utilizadas para caracterizar a população do estudo. A avaliação do estresse ocupacional ocorreu a partir do cálculo de um escore médio geral e escore médio para cada item da escala, identificando os estressores mais presentes, segundo a percepção dos profissionais. Os indicadores da EET variam de 1 a 5 e, quanto maior a média, maior o estresse. Consideram-se indicadores de níveis importantes de estresse os valores médios iguais ou superiores a 2,5 .

Realizou-se o cálculo dos escores das dimensões da UWES seguindo o modelo estatístico proposto no Manual Preliminar UWES - Utrecht Work Engagement Scale ${ }^{(16)}$, apresentando coeficiente de confiabilidade, valor mínimo, máximo, mediana, média ( \pm desvio padrão) e intervalo de confiança de $95 \%$ para cada dimensão da escala. Após o cálculo dos escores, realizou-se a interpretação dos valores de acordo com a decodificação do Manual Preliminar UWES, conforme mostra a Tabela I.

Tabela I - Modelo para interpretação dos valores dos escores médios da Utrecht Work Engagement Scale - UWES. São José do Rio Preto, 2019.

Classificação

Muito baixo

Baixo
Frequência dos sentimentos em relação ao trabalho (questões UWES)

0 a $0,99=1$ (Algumas vezes por ano)

1 a $1,99=2$ (Uma vez ou menos por mês)

2 a 2,99 = 3 (Algumas vezes por mês)

Médio

3 a $3,99=4$ (Uma vez por semana)

4 a $4,99=5$ (Algumas vezes por semana)

Muito alto

5 a $6=6$ (Todos os dias)

Aferiu-se a confiabilidade das medidas dos construtos da UWES pelo indicador de consistência interna alfa de Cronbach. Por fim, realizou-se a análise de correlação entre estresse ocupacional e as dimensões da UWES (Dedicação, Absorção, Vigor e Escore geral), utilizando-se o teste de correlação de Pearson, com nível de significância de $95 \%(p<0,05)$. Considerou-se correlação fraca para valores de $r$ até 0,30 , moderada para valores entre 0,40 e 0,60 , e forte para valores maiores que 0,70 .

Este estudo obteve a aprovação do Comitê de Ética em Pesquisa da Faculdade de Medicina de São José do Rio Preto (FAMERP), com Parecer n. ${ }^{\circ}$ 1.776.737.

\section{RESULTADOS}

Participaram do estudo 31 profissionais que responderam aos instrumentos, sendo 20 (64,5\%) cirurgiõesdentistas e 11 (35,5\%) ASB. Conforme mostra a Tabela II, houve prevalência do sexo feminino $(67,7 \%)$, faixa etária de 40 anos ou mais $(45,2 \%)$, casados $(83,9 \%)$, concursados/estatutários $(51,6 \%)$, com carga horária semanal de 40 horas $(74,2 \%)$, sem outra atividade remunerada $(61,3 \%)$, tempo de atuação na APS de três a dez anos $(41,9 \%)$ e satisfeitos com a profissão $(90,3 \%)$. 
Tabela II - Características sociodemográficas dos profissionais de saúde bucal da Atenção Primária à Saúde. São José do Rio Preto, 2019.

\begin{tabular}{|c|c|c|}
\hline Variáveis & $\mathbf{n}$ & $\%$ \\
\hline \multicolumn{3}{|l|}{ Área de atuação } \\
\hline Cirurgião-dentista & 20 & 64,5 \\
\hline Auxiliar de saúde bucal & 11 & 35,5 \\
\hline \multicolumn{3}{|l|}{ Sexo } \\
\hline Masculino & 10 & 32,3 \\
\hline Feminino & 21 & 67,7 \\
\hline \multicolumn{3}{|l|}{ Faixa etária } \\
\hline De 18 a 28 anos & 3 & 9,7 \\
\hline De 29 a 39 anos & 13 & 41,9 \\
\hline Com 40 anos ou mais & 14 & 45,2 \\
\hline Não respondeu & 1 & 3,2 \\
\hline \multicolumn{3}{|l|}{ Estado civil } \\
\hline Casado & 26 & 83,9 \\
\hline Solteiro & 3 & 9,7 \\
\hline Divorciado/Separado & 1 & 3,2 \\
\hline Não respondeu & 1 & 3,2 \\
\hline \multicolumn{3}{|l|}{ Regime de trabalho } \\
\hline Concursado (Estatutário) & 16 & 51,6 \\
\hline Contratado (Celetista) & 15 & 48,4 \\
\hline \multicolumn{3}{|l|}{ Carga horária semanal } \\
\hline 20 horas & 8 & 25,8 \\
\hline 40 horas & 23 & 74,2 \\
\hline \multicolumn{3}{|c|}{ Outra atividade remunerada } \\
\hline Sim & 11 & 35,5 \\
\hline Não & 19 & 61,3 \\
\hline Não respondeu & 1 & 3,2 \\
\hline \multicolumn{3}{|l|}{ Renda familiar ${ }^{*}$} \\
\hline De 2 a 5 salários & 15 & 48,4 \\
\hline De 6 a 10 salários & 5 & 16,1 \\
\hline Mais de 10 salários & 9 & 29,0 \\
\hline Não respondeu & 2 & 6,5 \\
\hline \multicolumn{3}{|l|}{ Horas diárias de sono } \\
\hline Menos de 6 horas & 5 & 16,1 \\
\hline De 6 a 8 horas & 26 & 83,9 \\
\hline \multicolumn{3}{|l|}{ Tempo atuação na APS } \\
\hline Até 2 anos & 5 & 16,1 \\
\hline De 3 a 10 anos & 13 & 41,9 \\
\hline Acima de 10 anos & 10 & 32,3 \\
\hline Não respondeu & 3 & 9,7 \\
\hline \multicolumn{3}{|l|}{ Pratica atividade física } \\
\hline Sim & 12 & 38,7 \\
\hline Não & 19 & 61,3 \\
\hline \multicolumn{3}{|c|}{ Satisfeito com a profissão/função } \\
\hline Sim & 28 & 90,3 \\
\hline Não & 3 & 9,7 \\
\hline \multicolumn{3}{|c|}{ Pensou em desistir da profissão/função } \\
\hline Sim & 4 & 12,9 \\
\hline Não & 27 & 87,1 \\
\hline
\end{tabular}

"Valor do salário mínimo: R\$937,00; APS: Atenção Primária à Saúde 
O escore geral de estresse ocupacional dos profissionais foi de 2,2 $(\mathrm{dp}= \pm 0,6$; mínimo $=1,0$ e máximo $=3,3$ ), evidenciando que a amostra não apresentou níveis importantes de estresse. No entanto sete itens obtiveram escores compatíveis com estresse importante $(\geq 2,5)$.

A Tabela III apresenta os escores para cada item da EET a partir da percepção dos profissionais de saúde bucal avaliados. Os estressores mais importantes foram: [Q2] O tipo de controle existente em meu trabalho me irrita $(2,8 ; \pm 1,0)$ [Q3] Falta de autonomia na execução do trabalho $(2,7 ; \pm 1,2)$; [Q13] Deficiência nos treinamentos para capacitação profissional $(2,7 ; \pm 1,1)$; [Q16] Falta de perspectivas de crescimento na carreira $(2,6 ; \pm 1,1)$; [Q1] Forma como as tarefas são distribuídas $(2,5 ; \pm 1,0)$; [Q15] Pouca valorização por superiores $(2,5 ; \pm 1,1)$; [Q22] Falta de tempo para realizar o trabalho $(2,5 ; \pm, 9)$.

Conforme apresentado na Tabela IV, os níveis de engajamento obtidos pelos profissionais de saúde bucal estudados variaram de 4,6 $( \pm 1,1)$ a $4,8( \pm 1,1)$, sendo classificados como altos em todas as dimensões da escala UWES. Os valores do coeficiente alfa de Cronbach variaram de 0,858 a 0,950, apontando consistência dos dados e confiabilidade dos resultados.

Estresse ocupacional e engajamento se correlacionaram negativamente. Conforme observado na Tabela V, a correlação entre estresse ocupacional e todas as dimensões da UWES foi fraca.

Tabela III - Distribuição dos escores para os itens da Escala de Estresse no Trabalho - EET, segundo a percepção dos profissionais de saúde bucal da Atenção Primária à Saúde. São José do Rio Preto, 2019.

Itens da Escala de Estresse no Trabalho - EET

Indicador geral

Q1 - A forma como as tarefas são distribuídas em minha área tem me deixado nervoso

Q2 - O tipo de controle existente em meu trabalho me irrita

Q3 - A falta de autonomia na execução do meu trabalho tem sido desgastante

Q4 - Tenho me sentido incomodado com a falta de confiança de meu superior sobre o meu trabalho

Q5 - Sinto-me irritado com a deficiência na divulgação de informações sobre decisões organizacionais

Q6 - Sinto-me incomodado com a falta de informações sobre minhas tarefas no trabalho

Q7 - A falta de comunicação entre mim e meus colegas de trabalho deixa-me irritado

Q8 - Sinto-me incomodado por meu superior tratar-me mal na frente de colegas de trabalho

Q9 - Sinto-me incomodado por ter que realizar tarefas que estão além de minha capacidade

Q10 - Fico de mau humor por ter que trabalhar durante muitas horas seguidas

Q11 - Sinto-me incomodado com a comunicação existente entre mim e meu superior

Q12 - Fico irritado com discriminação/favoritismo no meu ambiente de trabalho

Q13 - Tenho me sentido incomodado com a deficiência nos treinamentos para capacitação profissional

Q14 - Fico de mau humor por me sentir isolado na organização

Q15 - Fico irritado por ser pouco valorizado por meus superiores

Q16 - As poucas perspectivas de crescimento na carreira têm me deixado angustiado

Q17 - Tenho me sentido incomodado por trabalhar em tarefas abaixo do meu nível de habilidade

Q18 - A competição no meu ambiente de trabalho tem me deixado de mau humor

Q19 - A falta de compreensão sobre quais são minhas responsabilidades neste trabalho tem causado irritação

Q20 - Tenho estado nervoso por meu superior me dar ordens contraditórias

Q21 - Sinto-me irritado por meu superior encobrir meu trabalho bem feito diante de outras pessoas

Q22 - O tempo insuficiente para realizar meu volume de trabalho deixa-me nervoso

Q23 - Fico incomodado por meu superior evitar me incumbir de responsabilidades importantes
Média $( \pm d p)$

$2,2( \pm 0,6)$

$2,5( \pm 1,0)$

$2,8( \pm 1,0)$

$2,7( \pm 1,2)$

$2,1( \pm 1,2)$

$2,4( \pm 0,7)$

$2,1( \pm 0,7)$

$2,1( \pm 0,9)$

$1,8( \pm 1,2)$

$2,2( \pm 1,0)$

$2,1( \pm 0,9)$

$2,0( \pm 0,9)$

$2,2( \pm 1,1)$

$2,7( \pm 1,1)$

$2,0( \pm 0,7)$

$2,5( \pm 1,1)$

$2,6( \pm 1,1)$

$2,1( \pm 0,9)$

$1,7( \pm 0,6)$

$2,2( \pm 1,0)$

$2,1( \pm 0,9)$

$1,8( \pm 0,8)$

$2,5( \pm 0,9)$

$1,9( \pm 0,7)$ 
Tabela IV - Avaliação dos níveis de engajamento dos profissionais de saúde bucal da Atenção Primária à Saúde. São José do Rio Preto, 2019.

\begin{tabular}{|c|c|c|c|c|c|c|c|}
\hline Dimensões UWES & Alfa de Cronbach & Min & Max & Md & Média $\pm d p$ & IC (95\%) & Interpretação \\
\hline Vigor & 0,897 & 3,0 & 6,0 & 5,0 & $4,8 \pm 1,0$ & $4,4-5,2$ & Alto \\
\hline Dedicação & 0,868 & 2,6 & 6,0 & 5,4 & $4,8 \pm 1,1$ & $4,4-5,3$ & Alto \\
\hline Absorção & 0,858 & 1,8 & 6,0 & 5,0 & $4,6 \pm 1,1$ & $4,2-5,0$ & Alto \\
\hline Escore geral & 0,950 & 2,8 & 6,0 & 5,1 & $4,7 \pm 1,0$ & $4,4-5,1$ & Alto \\
\hline
\end{tabular}

Min: mínimo, Max: máximo, Md: mediana, dp: desvio padrão, IC 95\%: intervalo de confiança de 95\%; Dimensões da Utrecht Work Engagement: dedicação, absorção, vigor e escore geral

Tabela $V$ - Correlações entre a Escala de Estresse no Trabalho (EET) e as dimensões da Utrecht Work Engagement (UWES). São José do Rio Preto, 2019.

\begin{tabular}{ccc}
\hline $\begin{array}{c}\text { Dimensões } \\
\text { UWES }\end{array}$ & EET & Valor-p \\
\hline Dedicação & $-0,227^{* *}$ & 0,114 \\
Absorção & $-0,134^{* *}$ & 0,240 \\
Vigor & $-0,371^{*}$ & 0,022 \\
Escore geral & $-0,254^{* *}$ & 0,087 \\
\hline
\end{tabular}

${ }^{*} p<0,05 ;{ }^{* *} p<0,01$; EET: Escala de Estresse no Trabalho; Dimensões da Utrecht Work Engagement: dedicação, absorção, vigor e escore geral

\section{DISCUSSÃO}

A prevalência de profissionais do sexo feminino, encontrada no presente estudo, é similar ao apontado por estudos relacionados ao tema no âmbito da saúde ${ }^{(17-19)}$ e está relacionada ao processo de feminização dos profissionais de saúde vinculados aos serviços de Atenção Primária a Saúde. Esse fenômeno pode estar associado à mudança econômica ocorrida no Brasil na década passada, impulsionando a mulher para o mercado de trabalho ${ }^{(20)}$.

O predomínio de casados corrobora estudo com trabalhadores da ESF de um município do Rio Grande do Sul(21). Esse resultado pode estar relacionado à prevalência de profissionais com idade superior a 40 anos.

A prevalência de concursados se contrapõe aos resultados de estudo realizado em 2001/2002, pelo Departamento de Atenção Básica do Ministério da Saúde (DAB/MS), que identificou cerca de 20 a 30\% dos trabalhadores da ESF com vínculo precário de trabalho ${ }^{(22)}$. Por outro lado, corroboram estudos realizados em Vitória, Espírito Santo ${ }^{(23)}$ e em Minas Gerais ${ }^{(2,24)}$. Os vínculos profissionais estáveis é um fator positivo, que contribui para o desempenho laboral do trabalhador, garantido melhor assistência aos usuários do serviço de saúde ${ }^{(2,23,24)}$. Além disso, a garantia de proteção social que o vínculo trabalhista estável gera evita sentimentos de instabilidade, insegurança e estresse do trabalhador ${ }^{(23)}$.

Embora o escore geral de estresse ocupacional dos profissionais estudados não tenha identificado nível importante de estresse, alguns aspectos inerentes ao processo de trabalho na ESF foram apontados como estressantes, corroborando a literatura que afirma que o estresse laboral é um fenômeno multifatorial que pode se desenvolver precocemente na carreira odontológica ${ }^{(25)}$.

O sentimento de estresse pela falta de autonomia e tipo de controle, apontado pelos trabalhadores da atual pesquisa, pode estar relacionado à questões culturais inerentes à atuação em consultório particular, no qual o profissional possui autonomia sobre todos os aspectos da sua prática profissional. Estudo realizado no Distrito Federal corrobora esses resultados ao identificar que a prática da atenção individual, a concepção do trabalho na ESF e a autonomia dos profissionais de saúde bucal restringem sua atuação junto à eSB ${ }^{(26)}$. A literatura corrobora a queixa dos profissionais deste estudo sobre a falta de tempo para realização do trabalho ${ }^{(24,27,28)}$. 
A falta de capacitação profissional, destacada pelos profissionais do presente estudo, é apontada pela literatura como fator gerador de estresse ${ }^{(24,29)}$. Logo, é imprescindível que se reflita sobre a necessidade de aprimorar o processo de capacitação dos profissionais das eSB, implementando ações que os qualifiquem para atender às atribuições gerais e específicas estabelecidas pela Política Nacional de Atenção Básica ${ }^{(11)}$.

Outra estratégia possível de melhoria da capacitação dos profissionais de saúde bucal é o aprimoramento das matrizes curriculares dos cursos de Odontologia, garantindo formação em promoção e proteção da saúde, prevenção de agravos, diagnóstico, tratamento, acompanhamento, reabilitação e manutenção da saúde individual e coletiva para todas as famílias, indivíduos e grupos específicos assistidos pela ESF(11).

Os níveis de engajamento apresentados pelos profissionais de saúde bucal corroboram os resultados de estudo realizado com cirurgiões-dentistas indianos ${ }^{(9)}$ e outros estudos, realizados no Brasil $^{(30-32)}$ e em Portugal ${ }^{(32)}$, com profissionais da APS. Estes estudos evidenciam que, apesar da presença de fatores estressantes, os profissionais estudados possuem energia, disposição, concentração, capacidade de esforço e persistência frente às dificuldades existentes no ambiente de trabalho. O engajamento foi conceituado como um fenômeno relativamente estável e a relação positiva dos profissionais de saúde bucal com o ambiente laboral como essencial, pois é diretamente proporcional à satisfação do paciente em relação ao atendimento odontológico ${ }^{(9)}$.

A correlação negativa entre estresse ocupacional e engajamento, embora fraca no atual estudo, mostra que o aumento de um reduz o outro. Como o engajamento está ligado a atitudes positivas relacionadas ao trabalho, estimular o engajamento individual e coletivo pode reduzir o estresse ocupacional e aumentar o envolvimento dos profissionais no trabalho, proporcionando bem-estar para as eSB da ESF, melhora da qualidade do atendimento prestado aos usuários e aumento da resolutividade, beneficiando os serviços da APS.

Os resultados do presente estudo permitem refletir sobre a importância de ambientes profissionais salubres, que proporcionem bem-estar aos trabalhadores e reduzam os riscos de estresse. Os profissionais sentem falta de capacitação profissional, fundamental para a melhoria da qualidade dos serviços prestados e aumento da resolutividade. A identificação de aspectos estressores permite o direcionamento de ações de prevenção do estresse e promoção da saúde. É necessário o desenvolvimento de novos estudos, que envolvam maior número de profissionais e aprofundem a discussão sobre essa temática, para subsidiar a criação de ações que promovam a saúde dos profissionais de saúde bucal inseridos nos serviços de APS.

\section{CONCLUSÃO}

Os trabalhadores investigados apresentaram altos níveis de engajamento; alguns aspectos relacionados ao trabalho causam estresse. Estresse ocupacional e engajamento tendem a ser inversamente proporcionais.

\section{CONFLITOS DE INTERESSE}

Não existem conflitos de interesses.

\section{CONTRIBUIÇÕES}

Jussara Rossi Castro e Luciano Garcia Lourenção contribuíram na elaboração e delineamento do estudo; aquisição, análise e interpretação de dados; redação e/ou revisão do manuscrito. Claudia Eli Gazetta, Albertina Gomes da Silva e Paula Canova Sodré contribuíram na análise e interpretação de dados; redação e/ou revisão do manuscrito.

Artigo extraído da dissertação de mestrado de Jussara Rossi Castro intitulada Estresse ocupacional e engajamento em profissionais de saúde bucal da Atenção Primária à Saúde. Faculdade de Medicina de São José do Rio Preto, Programa de Pós-graduação em Psicologia e Saúde, 2019, 49 páginas.

\section{REFERÊNCIAS}

1. Nunes BP, Flores TR, Garcia LP, Chiavegatto ADP Filho, Thumé E, Facchini LA. Time trend of lack of access to health services in Brazil, 1998-2013. Epidemiol Serv Saúde [Internet]. 2016 [acesso em 2019 Set 29];25(4):777-87. doi: https://doi.org/10.5123/S1679-49742016000400011

2. Mattos GCM, Ferreira EF, Leite ICG, Greco RM. A inclusão da equipe de saúde bucal na Estratégia 
Saúde da Família: entraves, avanços e desafios. Cienc Saude Colet [Internet]. 2014 [acesso em 2019 Set 29];19(2):373-82. doi: https://dx.doi.org/10.1590/1413-81232014192.21652012

3. Ministério da Saúde (BR). Portaria $n^{\circ} 2.436$, de 21 de setembro de 2017. Aprova a Política Nacional de Atenção Básica, estabelecendo a revisão de diretrizes para a organização da Atenção Básica, no âmbito do Sistema Único de Saúde (SUS) [Internet]. Brasília: Ministério da Saúde; 2017 [acesso em 2019 Fev 07]. Disponível em: http://bvsms.saude.gov.br/bvs/saudelegis/gm/2017/prt2436_22_09_2017.html

4. Ferrari DA, Camarotto JA, Santos JEG. A atividade laboral do cirurgião dentista sob o contexto multidisciplinar da ergonomia: uma revisão sobre os domínios a serem considerados em ambiente odontológico. Rev Gest Prod Operações Sist [Internet]. 2016 [acesso em 2019 Set 30];11(1):159-77. doi: https://dx.doi.org/10.15675/gepros.v11i1.1348

5. Rowe DEO, Bastos AVB, Pinho APM. Múltiplos comprometimentos com o trabalho e suas influências no desempenho: um estudo entre professores do ensino superior no Brasil. Organ Soc [Internet]. 2013 [acesso em 2019 Fev 07];20(66):501-21. doi: http://dx.doi.org/10.1590/S1984-92302013000300008

6. Fan Y, Zheng Q, Liu S, Li Q. Construction of a new model of job engagement, psychological empowerment and perceived work environment among Chinese registered nurses at four large university hospitals: implications for nurse managers seeking to enhance nursing retention and quality of care. J Nurs Manag [Internet]. 2016 [acesso em 2019 Fev 07];24:646-55. doi: https://dx.doi.org/10.1111/jonm.12369

7. Sonnentag S. A task-level perspective on work engagement: A new approach that helps to differentiate the concepts of engagement and burnout. Burnout Res [Internet]. 2017 [acesso em 2019 Set 30];5(1):12-20. doi: https://doi.org/10.1016/j.burn.2017.04.001

8. Pauli J, Tomasi M, Gallon S, Coelho E. Satisfaction, conflict and engagement at work for high school teachers. Rev Pensamento Contemporâneo Adm [Internet]. 2017 [acesso em 2019 Set 30];11(4):72-85. doi: http://dx.doi.org/10.12712/rpca.v11i4.1004

9. Mukkavilli M, Kulkarni S, Doshi D, Reddy S, Reddy P, Reddy S. Assessment of work engagement among dentists in Hyderabad. Work [Internet]. 2018 [acesso em 2019 Fev 07];58(3):333-40. doi: https://doi. org/10.3233/WOR-172630

10. Brake HT, Bouman AM, Gorter R, Hoogstraten J, Eijkman M. Professional burnout and work engagement among dentists. Eur J Oral Sci [Internet]. 2007 [acesso em 2019 Fev 07];115(3):180-85. doi: https://doi. org/10.1111/j.1600-0722.2007.00439.x

11. Ministério da Saúde (BR), Secretaria de Vigilância em Saúde, Secretaria de Atenção à Saúde. Política Nacional de Promoção da Saúde: revisão da Portaria MS/GM nº 687, de 30 de março de 2006 [Internet]. Brasília: Ministério da Saúde; 2014 [acesso em 2018 Out 13]. Disponível em: http://bvsms.saude.gov.br/bvs/ publicacoes/pnps_revisao_portaria_687.pdf

12. Instituto Brasileiro de Geografia e Estatística. [População de] São José do Rio Preto [Internet]. [2019] [acesso em 2018 Out 13]. Disponível em: https://cidades.ibge.gov.br/brasil/sp/sao-jose-do-rio-preto/panorama

13. Prefeitura de São José do Rio Preto, Secretaria Municipal de Saúde. Painel de Monitoramento 2017: indicadores de 2016 [Internet]. 2017 [acesso em 2018 Out 13]. Disponível em: http://gestao.saude.riopreto. sp.gov.br/transparencia/arqu/painmoni/painel-monitoramento-2017.pdf

14. Paschoal T, Tamayo A. Validação da Escala de Estresse no Trabalho. Estud Psicol [Internet]. 2004 [acesso em 2019 Fev 13];9(1):45-52. Disponível em: http://www.scielo.br/pdf/epsic/v9n1/22380.pdf

15. Vazquez ACS, Magnan ES, Pacico JC, Hutz CS, Schaufeli WB. Adaptation and validation of the brazilian version of the Utrecht Work Engagement Scale. Psico-USF [Internet]. 2015 [acesso em $2019 \mathrm{Fev}$ 13];20(2):207-17. Disponível em: http://www.scielo.br/pdf/pusf/v20n2/1413-8271-pusf-20-02-00207.pdf

16. Agnst R, Benevides-Pereira AMT, Porto-Martins PC. Utrecht Work Engagement Scale. Curitiba: GEPEB; 2009.

17. Lourenção LG, Silva AG, Borges MA. Engagement in primary health care professionals. Esc Anna Nery Rev Enferm [Internet]. 2019 [acesso em 2019 Set 30];23(3):e20190005. doi: https://doi.org/10.1590/2177-9465EAN-2019-0005 
18. Monteiro-Marin J, Zubiaga F, Cereceda M, Demarzo MMP, Trenc P, Garcia-Campayo J. Burnout subtypes and absence of self-compassion in primary health care professionals: A cross-sectional study. PLoS ONE [Internet]. 2016 [acesso em 2019 Set 30]; 11(6):e0157499. doi: https://dx.doi.org/10.1371/journal. pone.0157499

19. Carlos MA. Perfil, qualidade de vida, estresse laboral, abuso e dependência de álcool dos trabalhadores da estratégia saúde da família [dissertação] [Internet]. Uberlândia: Universidade Federal de Uberlândia; 2017 [acesso em 2019 Fev 07]. Disponível em: https://repositorio.ufu.br/handle/123456789/19069

20. Guimarães NA. A igualdade substantiva e os novos desafios nas relações de gênero no trabalho. Rev Estud Fem [Internet]. 2016 [acesso em 2019 Set 30];24(2):639-43. doi: http://dx.doi.org/10.1590/1805-95842016v24n2p639

21. Moreira IJB, Horta JA, Duro LN, Borges DT, Cristofari AB, Chaves J, et al. Perfil sociodemográfico, ocupacional e avaliação das condições de saúde mental dos trabalhadores da Estratégia Saúde da Família em um município do Rio Grande do Sul, RS. Rev Bras Med Fam Comunidade [Internet]. 2016 [acesso em 2019 Set 30];11(38):1-12. doi: http://dx.doi.org/10.5712/rbmfc11(38)967

22. Ministério da Saúde (BR), Departamento de Gestão da Educação na Saúde. PCCS - SUS: diretrizes nacionais para a instituição de planos de carreiras, cargos e salários no âmbito do Sistema Único de Saúde [Internet]. Brasília: Ministério da Saúde; 2006 [acesso em 2019 Fev 07]. Disponível em: http://bvsms.saude. gov.br/bvs/publicacoes/pccs_diretrizes_nacionais_planos_carreiras_sus.pdf

23. Fleger DS, Guerra MA, Espindula KD, Lima RCD. O trabalho dos profissionais de saúde na estratégia saúde da família: relações complexas e laços institucionais frágeis. UFES Rev Odontol [Internet]. 2008 [acesso em 2019 Fev 07];10(4):17-23. Disponível em: http://periodicos.ufes.br/RBPS/article/viewFile/454/318

24. Lourenço EC, Silva ACB, Meneghin MC, Pereira AC. A inserção de equipes de saúde bucal no Programa Saúde da Família no Estado de Minas Gerais. Cienc Saude Colet [Internet]. 2009 [acesso em 2019 Fev 07];14(Supl 1):1367-77. doi: http://dx.doi.org/10.1590/S1413-81232009000800009

25. Singh P, Aulak DS, Mangat SS, Aulak MS. Systematic review: factors contributing to burnout in dentistry. Occup Med [Internet]. 2016 [acesso em 2019 Fev 07];66(1):27-31. doi: https://doi.org/10.1093/occmed/kqv119

26. Reis WG, Scherer MDA, Carcereri DL. O trabalho do Cirurgião-Dentista na Atenção Primária à Saúde: entre o prescrito e o real. Saúde Debate [Internet]. 2015 [acesso em 2019 Out 01];39(104):56-64. doi: https://doi. org/10.1590/0103-110420151040608

27. Moreira RS, Moraes MCL. Logros y dificultades del equipo de salud bucal en la estrategia de salud de familia en Brasil. Rev Fac Cienc Méd (Quito) [Internet]. 2017 [acesso em 2019 Out 01];41(2):19-25. Disponível em: http://200.12.169.32/index.php/CIENCIAS_MEDICAS/article/view/1515/1462

28. Costa DS, Rocha MP. O cirurgião-dentista e o mercado de trabalho no brasil: uma revisão sistemática da literatura. Id on Line Rev Multidiscip Psicol [Internet]. 2017 [acesso em 2019 Out 01];11(38):102-14. Disponível em: https://idonline.emnuvens.com.br/id/article/view/883/1252

29. Brigola S, Flores MT, Bordin D, Martins AS, Moimaz SAS, Fadel CB. Trabalho do cirurgião-dentista no serviço público de saúde e implicações sobre o estresse. Rev APS [Internet]. 2018 [acesso em 2019 Out 01];21(3):428-36. doi: https://doi.org/10.34019/1809-8363.2018.v21.16386

30. Silva AG. Níveis de engagement em enfermeiros da atenção primária à saúde [dissertação] [Internet]. São José do Rio Preto: Faculdade de Medicina de São José do Rio Preto; 2018 [acesso em 2019 Fev 07]. Disponível em: http://bdtd.famerp.br/handle/tede/443

31. Cordioli DFC, Cordioli JR Jr, Gazzeta CE, Silva AG, Lourenção LG. Occupational stress and work engagement in primary health care workers. Rev Bras Enferm [Internet]. 2019 [acesso em 2019 Fev 07];72(6):1580-7. Disponível em: http://www.scielo.br/scielo.php?pid=S0034$71672019000601580 \&$ script=sci_arttext

32. Cunha S, Gama C, Fevereiro M, Vasconcelos A, Sousa S, Neves AC, et al. A felicidade e o engagement no trabalho nos cuidados de saúde primários. Rev Port Med Geral Fam [Internet]. 2018 [acesso em 2019 Out 02];34(1):26-32. Disponível em: http://www.scielo.mec.pt/scielo.php?script=sci_arttext\&pid=S2182$51732018000100004 \&$ Ing $=p t$ 


\section{Endereço para correspondência:}

Luciano Garcia Lourenção

Universidade Federal do Rio Grande - FURG

Rua General Osório, s/n - Campus da Saúde

Bairro: Centro

CEP: 96200-400 - Rio Grande - RS - Brasil

E-mail: lucianolourencao.enf@gmail.com

\section{Endereço do primeiro autor:}

Jussara Rossi Castro

Faculdade de Medicina de São José do Rio Preto - FAMERP

Av. Brigadeiro Faria Lima, 5416

Bairro: Vila São Pedro

CEP: 15090-000 - São José do Rio Preto - SP - Brasil

E-mail: jussara-castroo@hotmail.com

Como citar: Castro JR, Gazetta CE, Silva AG, Sodré PC, Lourenção LG. Estresse ocupacional e engajamento em profissionais da atenção primária à saúde. Rev Bras Promoç Saúde. 2019;32:9157. 